\title{
Plasmodium 18S rRNA of intravenously administered sporozoites does not persist in peripheral blood
}

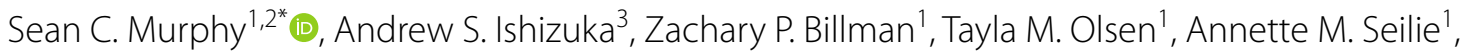
Ming Chang ${ }^{1}$, Nahum Smith ${ }^{1}$, Vorada Chuenchob ${ }^{4}$, Sumana Chakravarty ${ }^{5}$, B. Kim Lee Sim ${ }^{5}$, Stefan H. I. Kappe ${ }^{4}$, Stephen L. Hoffman ${ }^{5}$ and Robert A. Seder ${ }^{3}$

\begin{abstract}
Background: Plasmodium $18 \mathrm{~S}$ rRNA is a biomarker used to monitor blood-stage infections in malaria clinical trials. Plasmodium sporozoites also express this biomarker, and there is conflicting evidence about how long sporozoitederived $18 \mathrm{~S}$ rRNA persists in peripheral blood. If present in blood for an extended timeframe, sporozoite-derived $18 \mathrm{~S}$ rRNA could complicate use as a blood-stage biomarker.

Methods: Blood samples from Plasmodium yoelii infected mice were tested for Plasmodium 18S rRNA and their coding genes (rDNA) using sensitive quantitative reverse transcription PCR and quantitative PCR assays, respectively. Blood and tissues from Plasmodium falciparum sporozoite (PFSPZ)-infected rhesus macaques were similarly tested.

Results: In mice, when P. yoelii sporozoite inoculation and blood collection were performed at the same site (tail vein), low level rDNA positivity persisted for 2 days post-infection. Compared to intact parasites with high rRNAto-rDNA ratios, this low level positivity was accompanied by no increase in rRNA-to-rDNA, indicating detection of residual, non-viable parasite rDNA. When P. yoelii sporozoites were administered via the retro-orbital vein and blood sampled by cardiac puncture, neither P. yoelii 185 rRNA nor rDNA were detected $24 \mathrm{~h}$ post-infection. Similarly, there was no $P$. falciparum $18 \mathrm{~S}$ rRNA detected in blood of rhesus macaques 3 days after intravenous injection with extremely high doses of PfSPZ. Plasmodium 18S rRNA in the rhesus livers increased by approximately 101-fold from 3 to 6 days post infection, indicating liver-stage proliferation.
\end{abstract}

Conclusions: Beyond the first few hours after injection, sporozoite-derived Plasmodium 185 rRNA was not detected in peripheral blood. Diagnostics based on $18 \mathrm{~S}$ rRNA are unlikely to be confounded by sporozoite inocula in human clinical trials.

Keywords: $18 \mathrm{~S}$ rRNA, SSU, Small subunit rRNA, Plasmodium, Molecular diagnostic

\section{Background}

Plasmodium parasites are the causative agents of human malaria. Infection begins when female Anopheles mosquitoes take a blood meal. During feeding, mosquitoes transmit sporozoite-stage parasites into the dermis. Sporozoites make their way to blood vessels and then

\footnotetext{
*Correspondence: murphysc@uw.edu

${ }^{1}$ Departments of Laboratory Medicine and Microbiology, University

of Washington, 750 Republican St., E630, Seattle, WA 98109, USA

Full list of author information is available at the end of the article
}

to the liver, where they develop over the next several days. The sporozoite and liver stage parasites are clinically silent. At the completion of the liver stage, parasites are released into the bloodstream and invade erythrocytes. The resulting cyclical infection of erythrocytes is responsible for all clinical disease. During the erythrocyte stage, parasites can be detected in whole blood using several diagnostic tests such as microscopy of Giemsastained blood smears, lateral flow rapid diagnostic tests for parasite antigens and nucleic acid tests (NATs). In general, NATs are more analytically sensitive than other

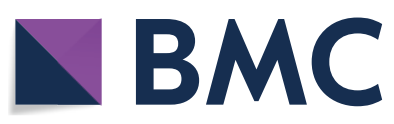

(c) The Author(s) 2018. This article is distributed under the terms of the Creative Commons Attribution 4.0 International License (http://creativecommons.org/licenses/by/4.0/), which permits unrestricted use, distribution, and reproduction in any medium, provided you give appropriate credit to the original author(s) and the source, provide a link to the Creative Commons license, and indicate if changes were made. The Creative Commons Public Domain Dedication waiver (http://creativecommons.org/ publicdomain/zero/1.0/) applies to the data made available in this article, unless otherwise stated. 
modalities [1]. The most common NAT targets are DNA genes encoding the Plasmodium $18 \mathrm{~S}$ ribosomal RNAs (hereafter called $18 \mathrm{~S}$ rDNA) or the $18 \mathrm{~S}$ rRNAs themselves, with testing by polymerase chain reaction (PCR) or reverse transcription PCR (RT-PCR), respectively [1].

Plasmodium 18S rRNA/rDNA-targeted NATs are intended to detect blood-stage parasites, but they also can detect the same sequences in sporozoite and liver stages. Most evidence suggests that sporozoites transit from the mosquito inoculation site to the liver in less than an hour [2]. However, if sporozoite-derived nucleic acids, or even sporozoites, continued to circulate for days following sporozoite exposure, these nucleic acids could generate false positive results for NATs intended to monitor for erythrocyte infection. An earlier study reported this type of NAT positivity in mice [3]. Abkallo and colleagues reported that Plasmodium yoelii $18 \mathrm{~S}$ rDNA was detectable in peripheral mouse blood by qPCR after $P$. yoelii sporozoite injection, but before emergence of infected erythrocytes from the liver at about $48 \mathrm{~h}$. Compared to Plasmodium falciparum, $P$. yoelii has a shorter liver stage with erythrocyte stage emergence $\sim 48 \mathrm{~h}$ post-inoculation. In the Abkallo study, CBA mice were infected with $2.5 \times 10^{4}$ P. yoelii sporozoites by tail vein injection, and blood was sampled by capillary action from the tail vein at later time points. For 90 min following $P$. yoelii sporozoite injection, $18 \mathrm{~S}$ rDNA was detected in tail vein peripheral blood at low and decreasing concentrations. $18 \mathrm{~S}$ rRNA was next detected $\sim 24 \mathrm{~h}$ postinjection (at $\sim 50-100$ copies of $18 \mathrm{~S} \mathrm{rDNA} / \mu \mathrm{L}$ of blood) followed by a decrease to baseline and then a marked rise at $48 \mathrm{~h}$ corresponding with erythrocyte stage infection and blood smear positivity. Based on these data, the authors concluded that NAT positivity prior to the emergence of erythrocyte stage parasites was from circulating pre-erythrocytic parasites. Such a result could complicate the use of Plasmodium 18S rRNA/rDNA NATs to assess infection in pre-clinical and clinical trials when using attenuated sporozoite vaccines that must be monitored for safety or using wild-type sporozoites for challenge studies [4]. Thus, to further investigate whether pre-erythrocytic parasites are a confounder of peripheral blood NATs, additional experiments using the $18 \mathrm{~S}$ rRNA biomarker were conducted in mice and non-human primates (NHP).

\section{Methods}

\section{Mice and Plasmodium yoelii infections}

Female BALB/cj mice (4-6 weeks old) were obtained from Jackson Laboratories (Barr Harbor, ME), housed in an IACUC-approved animal facility and used under an IACUC-approved protocol. Wild-type P. yoelii 17XNL sporozoites were obtained by salivary gland dissection from Anopheles stephensi mosquitoes reared at the Center for Infectious Disease Research (CID Research, Seattle, WA). Plasmodium yoelii sporozoites were injected intravenously in a $100-150 \mu \mathrm{L}$ volume via tail vein injection or by retro-orbital injection as noted in "Results" section. Parasites were purified using the accudenz gradient method [5] with minor modifications as reported [6]. Dried blood spots were collected by tail vein bleeds from alive mice (5-10 $\mu \mathrm{L}$ per spot) using a site in the distal tail usually $1-2 \mathrm{~cm}$ from the injection site. Venous whole blood was collected by cardiac puncture in euthanized mice as noted in the Results section. Venous whole blood samples were preserved in NucliSENS lysis buffer (bioMérieux) immediately after collection using a ratio of $50 \mu \mathrm{L}$ of blood to $2 \mathrm{~mL}$ of lysis buffer. DBS from tail vein collections were dried and desiccated. All preserved samples were stored at $-80^{\circ} \mathrm{C}$ until extraction.

Non-human primate and Plasmodium falciparum infections Wild-type PfSPZ were freshly dissected and purified at Sanaria, Inc. (Rockville, MD) and transported for $20 \mathrm{~min}$ to the IACUC-inspected NHP facility. Four rhesus macaques were intravenously infected with $6.5 \times 10^{6}$ PfSPZ under an IACUC-approved NHP protocol. Three or 6 days later, the animals were humanely euthanized and liver, lung, spleen and EDTA-anticoagulated whole blood samples were collected. Tissues were snap frozen in liquid nitrogen ( $250 \mathrm{mg}$ ) and whole blood was immediately preserved in lysis buffer using a ratio of $50 \mu \mathrm{L}$ of blood to $2 \mathrm{~mL}$ of lysis buffer. All preserved samples were stored at $-80{ }^{\circ} \mathrm{C}$ until extraction; frozen samples were shipped on dry ice. At the time of extraction, snap frozen tissues were emulsified in lysis buffer by bead beating using a ratio of $250 \mathrm{mg}$ of tissue to $10 \mathrm{~mL}$ of lysis buffer. Tissues/blood were also obtained from uninfected animals (called 'pre-inoculation').

\section{Nucleic acid extraction}

Total nucleic acids were extracted from mouse whole blood $(50 \mu \mathrm{L})$ and mouse dried blood spots $(\sim 5-10 \mu \mathrm{L})$ on an EasyMag instrument (bioMérieux) and eluted in a volume of $53 \mu \mathrm{L}$ as described [7]. At the time of processing, DBS were laser-cut as reported [8]. NHP blood $(50 \mu \mathrm{L})$ and tissues (derived from $50 \mathrm{mg}$ tissue equivalents from emulsified lysates of $50 \mathrm{mg}$ or larger $250 \mathrm{mg}$ samples, see Results) samples were extracted on an Abbott m2000sp and eluted in a volume of $53 \mu \mathrm{L}$ as described [8].

\section{qRT-PCR and qPCR}

Quantitative reverse transcription PCR (qRT-PCR) for $P$. yoelii $18 \mathrm{~S}$ rRNA was performed using primers/ 
probes and conditions as reported [6] using $5 \mu \mathrm{L}$ of template. qRT-PCR for P. falciparum was performed using primers, probes and conditions as reported [8] on an Abbott m2000rt using $15 \mu \mathrm{L}$ of template or BioRad CFX1000 using $5 \mu \mathrm{L}$ of template. With the exception of the DNA:RNA assay reported in Fig. 1, qRT-PCR results were calibrated against an absolute RNA standard curve to determine exact copy number values. For the rRNA:rDNA experiment in Fig. 1, absolute quantification was not possible because the DBS input volume varied from 5 to $10 \mu \mathrm{L}$ per spotinstead cycle thresholds $\left(\mathrm{C}_{\mathrm{T}}\right)$ were used. To analyse DNA only, the RT enzyme was omitted and quantitative PCR (qPCR) was performed; to analyse RNA, the RT enzyme was included but DNase was not used since rRNA is 1000-10,000 times more abundant than rDNA in intact parasites, depending on the assay and parasite species $[7,8]$. In mouse blood samples, detection of 18S rRNA was defined as a two-fold or greater increase in $18 \mathrm{~S}$ rRNA versus $18 \mathrm{~S}$ rDNA.

\section{Results}

Plasmodium yoelii 18S rRNA/rDNA detected when injection and sampling are both via tail vein reflects non-viable, residual parasite $18 \mathrm{~S}$ rDNA

To mimic the aforementioned Abkallo study, BALB/cj mice were injected in the tail vein with $1 \times 10^{4} \mathrm{P}$. yoelii sporozoites and were repeatedly sampled from the tail vein thereafter. RT-PCR and PCR generated positive albeit late $\mathrm{C}_{\mathrm{T}} \mathrm{s}$ from tail vein samples for all samples collected thereafter (Fig. 1a). However, even immediately after injection, the $\log _{10}$ difference in $18 \mathrm{~S}$ rRNA versus $18 \mathrm{~S}$ rDNA was much less than is measured in intact sporozoites. Like infected erythrocytes, intact $P$. yoelii sporozoites express much higher 18S rRNA concentrations than their coding rDNAs ( $>1000$-fold higher; Fig. 1b, spz bar). In contrast, the difference in $18 \mathrm{~S}$ rRNA versus $18 \mathrm{~S}$ rDNA in samples post-P. yoelii sporozoite injection was only tenfold higher immediately after injection, suggesting an increased contribution of $18 \mathrm{~S}$ rDNA. Although absolute quantification was not performed for this experiment, the estimated number of sporozoites detected per uL of mouse blood during these timepoints based on the laboratory's experience is estimated to be no more than 1-2 spz per uL (S. Murphy, pers. commun.). By $1 \mathrm{~h}$ post-inoculation, there was no difference in RT-PCR versus PCR $\mathrm{C}_{\mathrm{T}} \mathrm{s}$ and the total amount of nucleic acid was much less than that required to constitute a single intact sporozoite per sample. Thus, the results indicate that only residual $18 \mathrm{~S}$ rDNA was detected beyond $1 \mathrm{~h}$ post-injection (Fig. 1 and Table 1 ). In this study, positive detection of $18 \mathrm{~S}$ rRNA was qualitatively defined as twofold or greater increase over $18 \mathrm{~S}$ rDNA for a given sample. With this definition, there was no $18 \mathrm{~S}$ rRNA detection beyond $30 \mathrm{~min}$ after injection. The high $\mathrm{C}_{\mathrm{T}} \mathrm{s}$ measured when samples were taken later than 30 min post-administration corresponded to quantities of $18 \mathrm{~S}$ rRNA/rDNA nucleic acids that would not be adequate to constitute a single intact parasite $[7,8]$. As expected, at the start of the mouse erythrocytic stage, the difference between 18S rRNA versus rDNA increased to $>10,000$-fold, consistent with the start of erythrocyte stage infection and a high per-parasite $18 \mathrm{~S}$ rRNA content for intact, viable parasites. These data demonstrate that detection of $P$. yoelii $18 \mathrm{~S}$ rDNA can occur in mice when
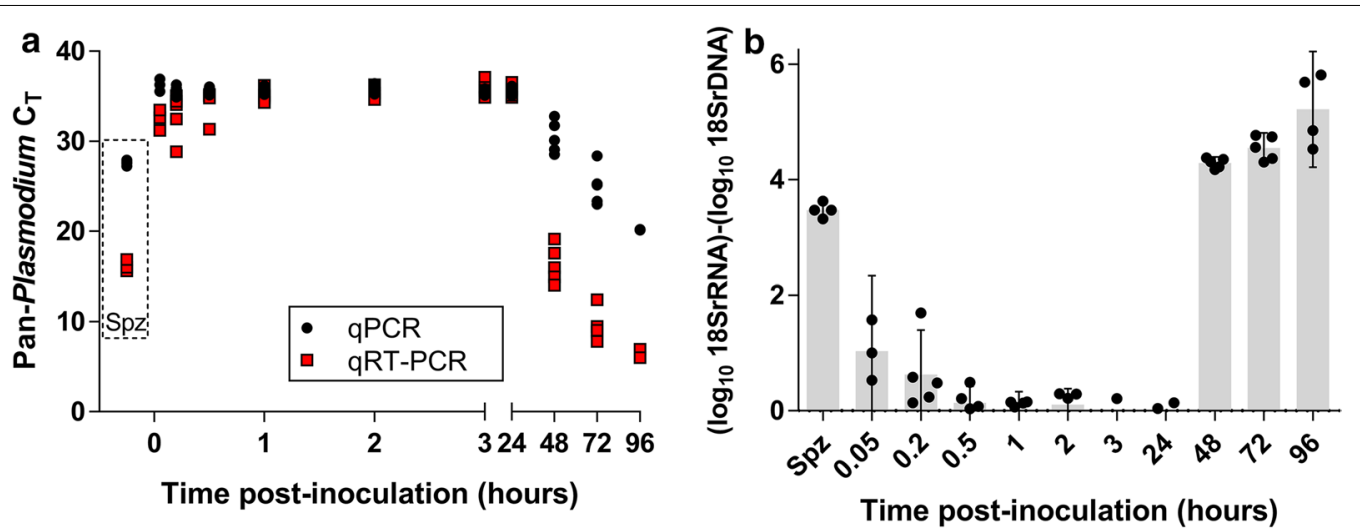

Fig. 1 Plasmodium yoelii 18S rRNA versus 18S rDNA in blood collected from the tail vein of BALB/cj mice after tail vein inoculation of sporozoites. a Plasmodium yoelii sporozoites were injected by the tail vein and then whole blood was collected onto dried blood spots at the indicated hour post-inoculation, preserved in lysis buffer and extracted for total nucleic acids. qPCR or qRT-PCR was performed and the $\mathrm{C}_{T} \mathrm{~s}$ are plotted. Red squares: qRT-PCR (18S rDNA + 18SrRNA), Black circles: qPCR (18S rDNA). Dashed box indicates $5 \times 10^{4}$ freshly-dissected sporozoites (Spz). Each data point represents a unique blood sample obtained from an individual animal at the stated time point. $\mathbf{b}$ Using the standard curve of diluted parasites, the $\log _{10}$ copies/mL difference between $18 \mathrm{~S}$ rRNA and 185 rDNA was calculated for each time point for each mouse 
Table 1 Summary of experiments to address route-specific Plasmodium 18S rRNA positivity in mouse studies

\begin{tabular}{|c|c|c|c|c|c|}
\hline Mouse studies & $\begin{array}{l}\text { 18S rRNA (\# } \\
\text { positive) }\end{array}$ & $18 \mathrm{~S}$ rRNA $C_{\mathrm{T}}$ (range) & $\begin{array}{l}\text { 18S rDNA (\# } \\
\text { positive) }\end{array}$ & $18 \mathrm{~S}$ rDNA $C_{T}$ (range) & $\Delta C_{\mathrm{T}}^{\mathbf{b}}$ (range) \\
\hline \multicolumn{6}{|c|}{ Tail vein inoculation/tail vein sampling experiment } \\
\hline Immediate & $3 / 3$ & $31.21-33.48$ & $3 / 3$ & $35.56-36.93$ & 2.08 to 5.73 \\
\hline $0.2 \mathrm{~h}$ & $3 / 5$ & $28.84-35.09$ & $5 / 5$ & $34.91-36.30$ & 0.81 to 6.18 \\
\hline $0.5 \mathrm{~h}$ & $1 / 5$ & $31.35-35.22$ & $5 / 5$ & $35.14-36.05$ & 0.14 to 3.79 \\
\hline $1 \mathrm{~h}$ & $0 / 5$ & $34.33-36.20$ & $5 / 5$ & $35.22-36.20$ & -0.99 to 1.03 \\
\hline $24 \mathrm{~h}$ & $0 / 5$ & $34.87-36.55$ & $5 / 5$ & $35.10-36.13$ & -0.42 to 0.84 \\
\hline $48 \mathrm{~h}$ & $5 / 5$ & $14.05-19.16$ & $5 / 5$ & $28.56-32.80$ & 13.64 to 14.51 \\
\hline \multicolumn{6}{|c|}{ Retro-orbital inoculation/cardiac puncture sampling experiment } \\
\hline $24 \mathrm{~h}$ & $0 / 3$ & N/A & $0 / 3$ & N/A & N/A \\
\hline
\end{tabular}

Tail vein (3-5 $\mu \mathrm{L}$ dried blood spots); cardiac puncture $(50 \mu \mathrm{L}$ whole blood)

N/A not applicable

a $18 \mathrm{~S}$ rRNA positivity defined as a $\geq 2$-fold relative increase in $18 \mathrm{~S}$ rRNA compared to rDNA

${ }^{b} \triangle C_{T}$ equals the $C_{T}$ for the $\mathrm{qPCR}$ (rDNA) minus the $\mathrm{C}_{\mathrm{T}}$ for the $\mathrm{qRT}-\mathrm{PCR}$ (rRNA) for an individual sample irrespective of positivity as described above such that a high $\Delta C_{T}$ indicates a large amount of $18 \mathrm{~S} r R N A$ relative to the coding DNA

P. yoelii sporozoites are inoculated via the tail vein and blood samples are likewise obtained from the tail vein.

\section{Circulating $P$. yoelii $18 \mathrm{~S}$ rRNA is not detected during pre-erythrocytic stages of infection in mice when injection and sampling sites are spatially distinct}

To eliminate sample site contamination as a factor, an experiment was performed wherein the P. yoelii sporozoite administration site and the blood sampling site were spatially separated. BALB/cj mice were retroorbitally injected with $2 \times 10^{4}$ wild-type $P$. yoelii sporozoites and then euthanized 1 day later and blood was collected by cardiac puncture. No $P$. yoelii $18 \mathrm{~S}$ rRNA was detected in $50 \mu \mathrm{L}$ whole blood samples $24 \mathrm{~h}$ after sporozoite inoculation ( $\mathrm{n}=3$ mice) (Table 1). All nucleic acid extractions and RT-PCR assays incorporated $P$. yoelii-infected blood positive controls and uninfected blood negative controls. These data demonstrate that $P$. yoelii $18 \mathrm{~S}$ rRNA is not detectable in peripheral blood $24 \mathrm{~h}$ post-administration when the injection and sampling site are spatially distinct.

\section{Lack of circulating $P$. falciparum $18 \mathrm{~S}$ rRNA in peripheral blood during pre-erythrocytic stages of infection in rhesus macaques}

To test whether $P$. falciparum $18 \mathrm{~S}$ rRNA circulates in larger animals, a NHP study was conducted in rhesus macaques. PfSPZ invade rhesus hepatocytes but with lower efficiency than human hepatocytes $[9,10]$, and rhesus do not support blood stage $P$. falciparum infections [11]. Four rhesus macaques (5 kg each; $250 \mathrm{~mL}$ circulatory volume) were intravenously infected with $6.5 \times 10^{6}$ freshly-dissected PfSPZ on day 0. Peripheral blood was collected immediately prior to inoculation. Two animals were euthanized on each of day 3 and 6 , and blood, liver, spleen and lung tissues were collected. Plasmodium falciparum 18S rRNA was not detected in the blood of any animal pre- (day 0 ) or post-inoculation (days 3 or 6 ) indicating that PfSPZ are cleared from the circulation within 3 days of injection (Fig. 2).

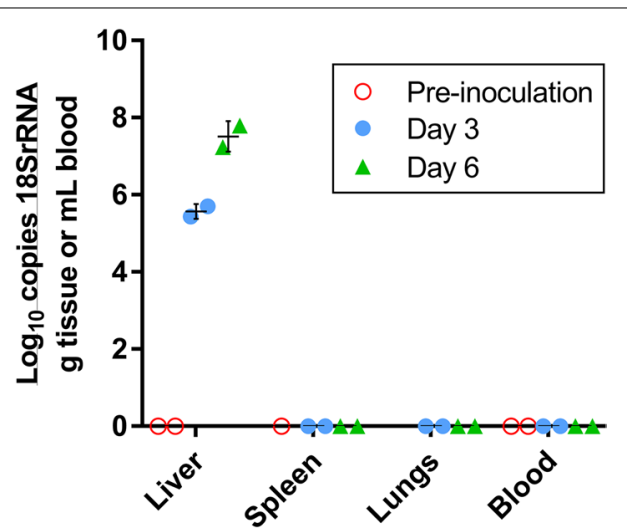

Fig. 2 Plasmodium falciparum 18S rRNA biomarker in rhesus macaques before and after high-dose PfSPZ inoculation. Tissue samples and whole blood were collected and preserved in lysis buffer as described. Nucleic acids were extracted and RT-PCR performed as described. The Plasmodium $18 \mathrm{~S}$ rRNA biomarker was detected only from liver samples. Open circles, pre-inoculation; closed circles, day 3 post-inoculation; closed triangles, day 6 post-inoculation. Bars show mean \pm standard deviation. Spleen was tested from only one uninfected animal, and lung samples were not tested from uninfected animals. Each data point represents a unique sample obtained from an individual animal at the stated time point 


\section{Accumulating $P$. falciparum 18S rRNA in the liver stage of rhesus macaques}

Tissue samples were also obtained from spleen, lung and liver in infected rhesus macaques. Plasmodium falciparum 18S rRNA was not detected in any of the lung or spleen samples three or 6 days post-infection. However, P. falciparum 18S rRNA was detected in liver samples from all animals after inoculation. Liver from an uninfected animal showed no such positivity. Samples for liver RT-PCR were derived from $\sim 250 \mathrm{mg}$ snap-frozen liver samples that were lysed and extracted for nucleic acids and subsequently tested by $18 \mathrm{~S}$ rRNA RT-PCR [8]. The P. falciparum $18 \mathrm{~S}$ rRNA liver burden of these animals was higher at day 6 post-infection compared to day 3 (Fig. 2). On day 3, the first two livers contained an average $3.9 \times 10^{5}$ copies per gram of $P$. falciparum $18 \mathrm{~S}$ rRNA. By day 6 , the mean Plasmodium $18 \mathrm{~S}$ rRNA copy number in the livers of the two remaining animals was $4.0 \times 10^{7}$ copies per gram of liver, an 101-fold increase compared to the mean copy number from the two livers collected on day 3 .

\section{Discussion}

The Plasmodium 18S rRNA/rDNA biomarker is a sensitive diagnostic marker that is able to achieve earlier detection of infection compared to blood smears [7, 12]. However, conflicting evidence about the persistence of sporozoite-derived 18S rRNA led to concerns about the potential for false positive results due to persistently circulating sporozoites or their byproducts.

Here, studies in mice demonstrated that $P$. yoelii sporozoite $18 \mathrm{~S}$ rRNA does not persistently circulate in peripheral blood and suggests that timing and concentration of the pre-erythrocytic $18 \mathrm{~S}$ rDNA positivity $24 \mathrm{~h}$ postchallenge in the Abkallo study may have been due to resampling of locally deposited contaminating $18 \mathrm{~S}$ rDNA. Direct data comparison between studies is limited since the assay used in the Abkallo study was a DNA-only test for Plasmodium $18 \mathrm{~S}$ rRNA genes. However, in agreement with that report, in this study low amounts of Plasmodium $18 \mathrm{~S}$ rRNA/rDNA could be detected. The rRNA versus rDNA comparison performed here suggests that this signal was likely from residual, locally deposited contaminating $P$. yoelii $18 \mathrm{~S}$ rDNA. Thus, the $18 \mathrm{~S}$ rRNA approach to parasite detection was advantageous since such tiny amounts of nucleic acid template do not constitute the $18 \mathrm{~S}$ rRNA content of a single parasite and would have been deemed negative by $18 \mathrm{~S}$ rRNA assays even if blood was collected from the tail vein site. In $50 \mu \mathrm{L}$ blood samples obtained distally from the inoculation site in mice, there was no evidence of peripheral circulation of $P$. yoelii sporozoite-derived 18S rRNA 1 day after administration. It is also notable that these cardiac puncture blood samples contained 10-20 times more volume of blood per sample than the tail vein-collected dried blood spots, further supporting the conclusion that there is no long lasting, peripherally circulating $P$. yoelii $18 \mathrm{~S}$ rRNA during the liver stage.

Studies in rhesus macaques also showed no evidence for persistent $P$. falciparum 18S rRNA circulation following administration of an exceptionally high dose of freshly-dissected PfSPZ. The PfSPZ dose given to NHP $\left(6.5 \times 10^{6}\right)$ was $>2000$-fold higher than the standard $3.2 \times 10^{3}$ PfSPZ of Sanaria ${ }^{\circledR}$ PfSPZ Challenge (aseptic, purified, cryopreserved PfSPZ) that always cause blood stage parasitemia in human subjects $[13,14]$, and comparably higher than the standard five mosquito bite dose used for controlled human malaria infections (CHMI) [15]. The NHP data support the conclusion that $P$. falciparum parasites and their $18 \mathrm{~S}$ rRNAs do not circulate in the days following $P$. falciparum sporozoite inoculation. These data, coupled with the relatively small number of sporozoites delivered by infected mosquito bites or by PfSPZ Challenge and the intended timing of diagnostic sample collection starting on day 6 or later, make the risk of sporozoite-induced false positives negligible in human challenge studies.

In addition, the NHP studies are consistent with expansion of liver-stage parasites (roughly 101-fold from day 3 to 6 post-infection) in these animals. While immunogenicity of PfSPZ vaccines has been assessed in rhesus [16], pre-erythrocytic protection studies are usually not tested in rhesus because they do not develop blood-stage $P$. falciparum infections after receiving $P$. falciparum sporozoites [11]. However, P. falciparum sporozoites invade many different cell types [17] including rhesus hepatocytes in vitro (albeit with lower efficiency than human hepatocytes) $[9,10]$. Plasmodium falciparum sporozoites that successfully invade rhesus hepatocytes in vitro subsequently express PfEXP1, a protein not expressed in sporozoites [9], indicating that the parasites continue to develop in these cells. Wild-type P. falciparum sporozoites in human hepatocytes proliferate $\sim 30,000$-fold during their 6.5 day development [2] with most proliferation occurring in the latter part of the cycle. The 101-fold increase observed from day 3 to 6 in rhesus macaques in this study demonstrates proliferation of P. falciparum in infected rhesus hepatocytes, though the current data does not match the proliferative potential of $P$. falciparum as measured in human liver. This study was limited by the small number of NHP ( $\mathrm{n}=2$ animals per timepoint) and a relatively small number of liver tissue samples overall. More systematic liver sampling using larger biopsies collected throughout the pre-erythrocytic stage could be useful for understanding and measuring the full growth potential of the P. falciparum liver stage in 
rhesus macaques. The data also suggest that rhesus could be further investigated for use in testing pre-erythrocytic stage-targeted vaccines and/or drugs using a liver-stage $P$. falciparum 18S rRNA endpoint as a measure of efficacy.

In agreement with the mouse and NHP findings presented herein, CHMI studies also support the lack of circulating $P$. falciparum sporozoite-derived $18 \mathrm{~S}$ rRNA after P. falciparum sporozoite inoculation. Plasmodium falciparum $18 \mathrm{~S}$ rRNA was not detected 7, 10 or 28 days after administration of genetically-attenuated GAP3KO sporozoites delivered by 150-200 P. falciparum GAP3KOinfected mosquito bites [18]. The GAP3KO study is highly relevant to the question of persistent circulating $18 \mathrm{~S}$ rRNA/rDNA since the attenuated parasite does not lead to formation of infected erythrocytes so any circulating $P$. falciparum $18 \mathrm{~S}$ rRNA would have been presumed to be $P$. falciparum sporozoite-derived.

Like the animal studies presented herein, rising biomarker positivity from day 6 to 7 post-infection in CHMI studies at multiple centres [7, 19-23] strongly supports the well-studied timing of erythrocyte stage emergence from the human liver and suggests that testing starting on days 6-7 post-inoculation is appropriate in human clinical trials. These findings are further corroborated by earlier studies aimed at measuring the duration of the liver stage by culturing parasites during days 5-9.5 post-inoculation [24] where $P$. falciparum could only be cultured from $P$. falciparum sporozoite-infected human volunteers from day 6.5 onward. In another early study, human volunteers were bitten by infected mosquitoes and their blood was then sub-inoculated into different human recipients at timepoints thereafter [2]. Plasmodium falciparum infections could only be successfully sub-inoculated into recipients when donor blood was collected within $1 \mathrm{~h}$ of the donor's original mosquito bites and not again until 5-6 days later when the erythrocyte stage had begun. The overall conclusion is that in the days that follow sporozoite exposure, sporozoites do not circulate in peripheral blood. Therefore, the data collectively indicate that in humans the presence of $P$. falciparum $18 \mathrm{~S}$ rRNA days after sporozoite inoculation reflects $P$. falciparum erythrocyte stage parasite emergence, not the persistence of $P$. falciparum sporozoite-derived $18 \mathrm{~S}$ rRNA.

\section{Conclusions}

In sum, $18 \mathrm{~S}$ rRNA is a sensitive marker of Plasmodium blood stage infection. Beyond the first few hours immediately after injection, sporozoite-derived Plasmodium $18 \mathrm{~S}$ rRNA does not persist in peripheral blood. Diagnostic tests based on 18S rRNA are unlikely to be confounded by sporozoite inocula.

\section{Abbreviations}

NHP: non-human primates; NAT: nucleic acid test; PfSPZ: P. falciparum sporozoite; PCR: polymerase chain reaction; qPCR: quantitative PCR; qRT-PCR: quantitative RT-PCR; RT-PCR: reverse transcription PCR; rDNA: ribosomal DNA; rRNA: ribosomal RNA.

\section{Authors' contributions}

SCM, TMO, ASI, ZPB, AMS, NS, and EC performed the experiments. SCM, SC, BKLS, SK, SLH and RAS provided reagents. SCM and MC wrote the manuscript. All authors reviewed the data. All authors read and approved the final manuscript.

\section{Author details \\ ${ }^{1}$ Departments of Laboratory Medicine and Microbiology, University of Wash- ington, 750 Republican St., E630, Seattle, WA 98109, USA. ${ }^{2}$ Center for Emerg- ing and Re-emerging Infectious Diseases, University of Washington, 750 Republican St., Seattle, WA 98109, USA. ${ }^{3}$ Vaccine Research Center, National Institute of Allergy and Infectious Diseases, National Institutes of Health, Building 40, Room 3512, 40 Convent Drive, Bethesda, MD 20814, USA. ${ }^{4}$ Center for Infectious Disease Research, 307 Westlake Ave N \#500, Seattle, WA 98109, USA. ${ }^{5}$ Sanaria, Inc., 9800 Medical Center Drive, Suite A209, Rockville, MD 20850, USA.}

\section{Acknowledgements}

We thank the staff of the Insectary at the Center for Infectious Disease Research for assistance with sporozoite culture.

\section{Competing interests}

The authors declare that they have no competing interests.

\section{Availability of data and materials}

All data generated or analysed during this study are included in this published article.

\section{Ethics approval and consent to participate}

Mouse studies were approved by the University of Washington Institutional Animal Care and Use Committee, and NHP studies were approved by the Institutional Animal Care and Use Committee of the Vaccine Research Center, $\mathrm{NIH}$. All animals were cared for in accordance with the NIH Guide for the Care and Use of Laboratory Animals.

\section{Funding}

Funding was from the Bill and Melinda Gates Foundation for Plasmodium molecular diagnostic test development (OPP1133622) and in part by the National Institutes of Health (AI-38858 and P30 Al027757). NHP work was supported by the Intramural Research Program of the US National Institutes of Health. Production of the PfSPZ by Sanaria Inc. was supported by NIAID Small Business Innovation Research Grants 4R44AI055229-08, 3R44AI055229-06S1, and 5R44AI058499-05.

\section{Publisher's Note}

Springer Nature remains neutral with regard to jurisdictional claims in published maps and institutional affiliations.

Received: 30 April 2018 Accepted: 19 July 2018

Published online: 27 July 2018

References

1. Murphy SC, Shott JP, Parikh S, Etter P, Prescott WR, Stewart VA. Malaria diagnostics in clinical trials. Am J Trop Med Hyg. 2013;89:824-39.

2. Fairley $\mathrm{NH}$. Sidelights on malaria in man obtained by subinoculation experiments. Trans Royal Soc Trop Med Hyg. 1947;40:621-76.

3. Abkallo HM, Liu W, Hokama S, Ferreira PE, Nakazawa S, Maeno Y, et al. DNA from pre-erythrocytic stage malaria parasites is detectable by PCR in the faeces and blood of hosts. Int J Parasitol. 2014;44:467-73. 
4. Richie TL, Billingsley PF, Sim BK, Epstein JE, Lyke KE, Mordmuller B, et al. Progress with Plasmodium falciparum sporozoite (PfSPZ)-based malaria vaccines. Vaccine. 2015;33(52):7452-61.

5. Kennedy M, Fishbaugher ME, Vaughan AM, Patrapuvich R, Boonhok R, Yimamnuaychok $\mathrm{N}$, et al. A rapid and scalable density gradient purification method for Plasmodium sporozoites. Malar J. 2012;11:421.

6. Billman ZP, Seilie AM, Murphy SC. Purification of Plasmodium sporozoites enhances parasite-specific CD8+T cell responses. Infect Immun. 2016:84:2233-42.

7. Murphy SC, Prentice JL, Williamson K, Wallis CK, Fang FC, Fried M, et al Real-time quantitative reverse transcription PCR for monitoring of bloodstage Plasmodium falciparum infections in malaria human challenge trials. Am J Trop Med Hyg. 2012;86:383-94.

8. Murphy SC, Daza G, Chang M, Coombs R. Laser cutting eliminates nucleic acid cross-contamination in dried-blood-spot processing. J Clin Microbiol. 2012;50:4128-30.

9. Epstein JE, Tewari K, Lyke KE, Sim BK, Billingsley PF, Laurens MB, et al. Live attenuated malaria vaccine designed to protect through hepatic CD8(+) T cell immunity. Science. 2011;334:475-80.

10. Millet $P$, Collins WE, Aikawa M, Cochrane AH, Nguyen-Dinh P. Use of non-human primate hepatocytes for in vitro study of the pre-erythrocytic stages of malaria parasites. Bull World Health Organ. 1990;68(Suppl):60-5.

11. Dolan SA, Proctor JL, Alling DW, Okubo Y, Wellems TE, Miller LH. Glycophorin B as an EBA-175 independent Plasmodium falciparum receptor of human erythrocytes. Mol Biochem Parasitol. 1994;64:55-63.

12. Tadesse FG, Lanke K, Nebie I, Schildkraut JA, Goncalves BP, Tiono AB, et al. Molecular markers for sensitive detection of Plasmodium falciparum asexual stage parasites and their application in a malaria clinical trial. Am J Trop Med Hyg. 2017;97:188-98.

13. Mordmuller B, Supan C, Sim KL, Gomez-Perez GP, Ospina Salazar CL, Held $J$, et al. Direct venous inoculation of Plasmodium falciparum sporozoites for controlled human malaria infection: a dose-finding trial in two centres. Malar J. 2015;14:117.

14. Gomez-Perez GP, Legarda A, Munoz J, Sim BK, Ballester MR, Dobano C, et al. Controlled human malaria infection by intramuscular and direct venous inoculation of cryopreserved Plasmodium falciparum sporozoites in malaria-naive volunteers: effect of injection volume and dose on infectivity rates. Malar J. 2015;14:306.

15. Chulay JD, Schneider I, Cosgriff TM, Hoffman SL, Ballou WR, Quakyi IA et al. Malaria transmitted to humans by mosquitoes infected from cultured Plasmodium falciparum. Am J Trop Med Hyg. 1986;35:66-8.

16. Ishizuka AS, Lyke KE, DeZure A, Berry AA, Richie TL, Mendoza FH, et al. Protection against malaria at 1 year and immune correlates following PfSPZ vaccination. Nat Med. 2016;22:614-23.

17. Amino R, Thiberge S, Martin B, Celli S, Shorte S, Frischknecht F, et al. Quantitative imaging of Plasmodium transmission from mosquito to mammal. Nat Med. 2006:12:220-4.

18. Kublin JG, Mikolajczak SA, Sack BK, Fishbaugher ME, Seilie A, Shelton L, et al. Complete attenuation of genetically engineered Plasmodium falciparum sporozoites in human subjects. Sci Transl Med. 2017;9:eaad9099.

19. Hodgson SH, Douglas AD, Edwards NJ, Kimani D, Elias SC, Chang M, et al. Increased sample volume and use of quantitative reverse-transcription PCR can improve prediction of liver-to-blood inoculum size in controlled human malaria infection studies. Malar J. 2015;14:33.

20. Roestenberg M, McCall M, Hopman J, Wiersma J, Luty AJ, van Gemert GJ, et al. Protection against a malaria challenge by sporozoite inoculation. $\mathrm{N}$ Engl J Med. 2009;361:468-77.

21. Roestenberg M, de Vlas SJ, Nieman AE, Sauerwein RW, Hermsen CC. Efficacy of preerythrocytic and blood-stage malaria vaccines can be assessed in small sporozoite challenge trials in human volunteers. J Infect Dis. 2012;206:319-23.

22. Roestenberg M, O'Hara GA, Duncan CJ, Epstein JE, Edwards NJ, Scholzen A, et al. Comparison of clinical and parasitological data from controlled human malaria infection trials. PLOS ONE. 2012;7:e38434.

23. Mordmuller B, Surat G, Lagler H, Chakravarty S, Ishizuka AS, Lalremruata $A$, et al. Sterile protection against human malaria by chemoattenuated PfSPZ vaccine. Nature. 2017:542:445-9.

24. Murphy JR, Baqar S, Davis JR, Herrington DA, Clyde DF. Evidence for a 6.5-day minimum exoerythrocytic cycle for Plasmodium falciparum in humans and confirmation that immunization with a synthetic peptide representative of a region of the circumsporozoite protein retards infection. J Clin Microbiol. 1989:27:1434-7.
Ready to submit your research? Choose BMC and benefit from:

- fast, convenient online submission

- thorough peer review by experienced researchers in your field

- rapid publication on acceptance

- support for research data, including large and complex data types

- gold Open Access which fosters wider collaboration and increased citations

- maximum visibility for your research: over $100 \mathrm{M}$ website views per year

At $\mathrm{BMC}$, research is always in progress.

Learn more biomedcentral.com/submissions 